Fachbereich Informatik

Lehrstuhl für Software-Technologie

MEM O Nr. 146

\title{
Factoring Stochastic Relations
}

Ernst-Erich Doberkat

Januar 2004

Internes Memorandum des

Lehrstuhls für Software-Technologie

Prof. Dr. Ernst-Erich Doberkat

Fachbereich Informatik

Universität Dortmund

Baroper Straße 301

D-44227 Dortmund

ISSN 0933-7725 


\title{
Factoring Stochastic Relations
}

\author{
Ernst-Erich Doberkat* \\ Chair for Software Technology \\ University of Dortmund \\ doberkat@acm.org
}

January 17, 2004

\begin{abstract}
When a system represented through a stochastic model is observed, the equivalence of behavior is described through the observation that equivalent inputs lead to equivalent outputs. This paper has a look at the systems that arise when the stochastic model is factored through the congruence. Congruences may refine each other, and we show that this refinement is reflected through factoring. We also show that factoring a factor does not give rise to any new constructions, since we are kept in the realm of factors for the original system. Thus we cannot have infinite long chains of factors, so that no new behavior can arise from the original system upon factoring (a system and its factors are bisimilar, after all).
\end{abstract}

\section{Introduction}

A stochastic relation $K: X \rightsquigarrow Y$ models the transformation of inputs to outputs with stochastic means; the special case of a state transition system is covered by assuming $X=$ $Y=S$ with $S$ as the state space. If the input to the system is $x \in X$, the subprobability measure $K(x)$ on $Y$ yields the distribution of the outputs, since $K(x)(Y)<1$ is not excluded, non-terminating processes are taken into account. A congruence for $K$ models equivalent behavior. This is modelled through a pair $\mathrm{c}=(\alpha, \beta)$ of equivalence relations $\alpha$ on $X$ and $\beta$ on $Y$ such that $\alpha$-equivalent inputs are transformed into $\beta$-equivalent outputs. We usually cannot address the outputs directly, $(K(x)(\{y\})$ may always be 0 in an uncountable space), hence we characterize equivalent behavior through $\beta$-invariant output sets: $B \subseteq Y$ is $\beta$-invariant if $\beta$ cannot distinguish the elements of $B$, formally, iff $y \in B$ and $y \beta y^{\prime}$ together imply $y^{\prime} \in B$. In a very natural way, this congruence induces a relation $K_{\mathrm{c}}$ on the set of equivalence classes. We will show that each congruence $d$ on that system may be represented up to isomorphism through a congruence on the original one, since $\left(K_{\mathrm{c}}\right)_{\mathrm{d}}$ is isomorphic to $K_{\mathrm{e}}$ for some congruence e which can be explicitly constructed from c and $d$. This is the stochastic analogon of Noether's Isomorphism Theorem for normal subgroups. Via the canonic projection it becomes clear that congruences are essentially the kernels of morphisms, so factoring of morphisms is investigated. It is shown that each morphism can be factored through a suitable isomorphism. This has as a consequence that the refinement of congruences can be represented through factoring.

\footnotetext{
${ }^{*}$ Research funded in part by Deutsche Forschungsgemeinschaft, grant DO 263/8-1, Algebraische Eigenschaften stochastischer Relationen
} 
All this can only be done when some assumptions on the probability spaces are made. In our case we assume that the input and the output space are analytic spaces, thus images under Borel measurable maps from Polish spaces, which in turn are complete and separable metric spaces. Analycity may sound rather exotic, but these spaces have some measure theoretic properties which make the necessary constructions possible: this class of spaces is preserved for example under forming factors of equivalence relations which are spawned by Borel measurable maps. This is the kind of relations we are working with.

Congruences were investigated first in [2] under the name of bisimulations when investigating the approximation of labelled Markov transition processes. The main example for these bisimulations came from modal logic, where two states are called equivalent iff they satisfy exactly the same formulas. Originally only logics were investigated that have a countable number of diamonds, in [6] this is generalized to general modal logic, and a theorem of Hennessy-Milner type is proved. On the other hand, when transition systems are modelled through coalgebras, then congruences arise in a natural way from bisimulations (in Milner's original definition), and vice versa, and from morphisms for coalgebras, as is discussed at length in [10]. The connection between congruences and bisimulations is not as close for stochastic relations as for coalgebras. This is mainly due to the fact that congruences are set-based constructions, and bisimulations are spans of morphisms: these entities can be transformed into each other in the set-based case on which coalgebras nearly always rest, but there is a crevice not easily bridged for stochastic relations (as can be seen when dealing with elementary constructions like the converse for the stochastic case).

The paper is organized as follows: we first give the necessary definitions and carry out some helpful auxiliary constructions in Section 2, Section 3 establishes two isomorphisms and draws some conclusions, Section 4 wraps it up and proposes some further work.

Acknowledgement Georgios Lajios provided some helpful comments.

\section{Stochastic Relations}

This section collects some basic facts from topology and measure theory for the reader's convenience and for later reference. It defines stochastic relations.

A Polish space $(X, \mathcal{G})$ is a topological space which is second countable, i.e., which has a countable dense subset, and which is metrizable through a complete metric. A measurable space $(X, \mathcal{A})$ is a set $X$ with a $\sigma$-algebra $\mathcal{A}$. The Borel sets $\mathcal{B}(X, \mathcal{G})$ for the topology $\mathcal{G}$ is the smallest $\sigma$-algebra on $X$ which contains $\mathcal{G}$. Given two measurable spaces $(X, \mathcal{A})$ and $(Y, \mathcal{B})$, a map $f: X \rightarrow Y$ is $\mathcal{A}-\mathcal{B}$-measurable whenever $f^{-1}[\mathcal{B}] \subseteq \mathcal{A}$ holds, where $f^{-1}[\mathcal{B}]:=$ $\left\{f^{-1}[B] \mid B \in \mathcal{B}\right\}$ is the set of inverse images $f^{-1}[B]:=\{x \in X \mid f(x) \in B\}$ of elements of $\mathcal{B}$. Note that $f^{-1}[\mathcal{B}]$ is a $\sigma$-algebra, provided $\mathcal{B}$ is one. If $f$ is $\mathcal{A}-\mathcal{B}$ - measurable and a bijection such that $f^{-1}: Y \rightarrow X$ is also $\mathcal{B}-\mathcal{A}$ - measurable, then $f$ is called a Borel isomorphism. A measurable space which is Borel isomorphic to a Polish space is again a Polish space. If the $\sigma$-algebras are the Borel sets of some topologies on $X$ and $Y$, resp., then a measurable map is called Borel measurable or simply a Borel map. The real numbers $\mathbb{R}$ carry always the Borel structure induced by the usual topology which will usually not be mentioned explicitly when talking about Borel maps.

If $(T, \mathcal{T})$ is a measurable space, and $f: T \rightarrow S$ is a map, then the final $\sigma$-algebra with respect to $f$ and $\mathcal{T}$ is the largest $\sigma$-algebra $\mathcal{S}$ on $S$ such that $f$ is $\mathcal{T}$-S-measurable. If $(R, \mathcal{R})$ is a

January 17, 2004 
measurable space, then a map $g: S \rightarrow R$ is $\mathcal{S}$ - $\mathcal{R}$-measurable iff $g \circ f$ is $\mathcal{T}$ - $\mathcal{R}$-measurable. This universal property characterizing the final $\sigma$-algebra will be helpful later.

An analytic set $X \subseteq Z$ for a Polish space $Z$ is the image $f[Y]$ of a Polish space $Y$ for some Borel measurable map $f: Y \rightarrow Z$. Endow $X$ with the trace $\mathcal{A}$ of $\mathcal{B}(Z)$ on $X$, i.e., $\mathcal{A}:=\{B \cap X \mid B \in \mathcal{B}(Z)\}$, the elements of which still being called Borel sets. A measurable space $\left(X^{\prime}, \mathcal{A}^{\prime}\right)$ which is Borel isomorphic to $(X, \mathcal{A})$ is called an analytic space. The elements of $\mathcal{A}^{\prime}$ are still called the Borel sets of $X^{\prime}, \mathcal{A}^{\prime}$ itself is denoted by $\mathcal{B}\left(X^{\prime}\right)$.

When the context is clear, we will write down topological or measurable spaces without their topologies and $\sigma$-algebras, resp., and the Borel sets are always understood with respect to the topology under consideration.

Denote for a measurable space $(T, \mathcal{T})$ by $\mathfrak{S}(T, \mathcal{T})$ the set of all subprobability measures on $(T, \mathcal{T})$ which is equipped with the weak ${ }^{*}-\sigma$-algebra for a measurable structure. The latter $\sigma$ algebra is the smallest $\sigma$-algebra on $\mathfrak{S}(X, \mathcal{A})$ which renders all maps $\mu \mapsto \mu(D)$ measurable, where $D \in \mathcal{T}$. It is well known that $\mathfrak{S}(T)$ with this $\sigma$-algebra is an analytic space, provided $T$ is one.

Definition 1 Given two analytic spaces $X$ and $Y$, a stochastic relation $K: X \rightsquigarrow Y$ between $X$ and $Y$ is a Borel map from $X$ to $\mathfrak{S}(Y)$.

Hence $K: X \rightsquigarrow Y$ is a stochastic relation from $X$ to $Y$ iff

1. $K(x)$ is a subprobability measure on $Y$ for all $x \in X$,

2. $x \mapsto K(x)(D)$ is a measurable map for each measurable set $D \subseteq Y$.

An $\mathcal{T}$ - $\mathcal{S}$ - measurable map $f: T \rightarrow S$ between the measurable spaces $(T, \mathcal{T})$ and $(S, \mathcal{S})$ induces a map $\mathfrak{S}(f): \mathfrak{S}(X, \mathcal{A}) \rightarrow \mathfrak{S}(Y, \mathcal{B})$ upon setting $\mathfrak{S}(f)(\mu)(D):=\mu\left(f^{-1}[D]\right)(\mu \in$ $\mathfrak{S}(T, \mathcal{T}), D \in \mathcal{S})$. It is easy to see that $\mathfrak{S}(f)$ is measurable.

The category Stoch has as objects stochastic relations $\mathrm{K}=(X, Y, K)$ for analytic spaces $X, Y$ and $K: X \rightsquigarrow Y$. A morphism $\mathrm{f}: \mathrm{K}_{1} \rightarrow \mathrm{K}_{2}$ between the objects $\mathrm{K}_{1}=\left(X_{1}, Y_{1}, K_{1}\right)$ and $\mathrm{K}_{2}=\left(X_{2}, Y_{2}, K_{2}\right)$ is a pair $\mathrm{f}=(\phi, \psi)$ of surjective measurable maps $\phi: X_{1} \rightarrow X_{2}$ and $\psi: Y_{1} \rightarrow Y_{2}$ such that $K_{1} \circ \phi=\mathfrak{S}(\psi) \circ K_{2}$ holds, i.e., such that the diagram

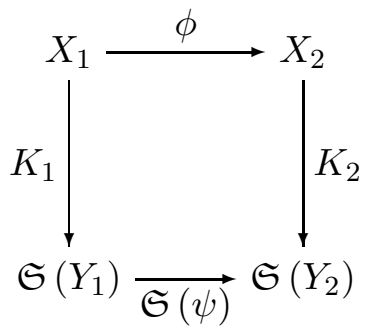

is commutative. Spelt out, this condition means that

$$
K_{2}\left(\phi\left(x_{1}\right)\right)\left(B_{2}\right)=K_{1}\left(x_{1}\right)\left(\psi^{-1}\left[B_{2}\right]\right)
$$

holds for each $x_{1} \in X_{1}$ and each Borel set $B_{2} \subseteq Y_{2}$.

Thus Stoch just is the comma category $1_{\mathbf{A}} \downarrow \mathfrak{S}[8$, Section II.6] with $\mathbf{A}$ as the category of analytic spaces with surjective measurable maps as morphisms, and $\mathfrak{S}: \mathbf{A} \rightarrow \mathbf{A}$ as the subprobability functor. Alternatively, $\mathfrak{S}$ can be seen as the functorial part of a monad (for 
this monad, the reader may wish to consult [7], for applications, see [3]). A subcategory of Stoch is the well-known category of Markov state transition systems. These systems may formally be described through a stochastic relation $K: S \rightsquigarrow S$ with $S$ as a state space, and $K$ modelling state transitions. A morphism $\phi:(S, K) \rightarrow\left(S^{\prime}, K^{\prime}\right)$ is a surjective Borel map $\phi: S \rightarrow S^{\prime}$ such that $K^{\prime} \circ \phi=\mathfrak{S}(\phi) \circ K$.

An equivalence relation $\rho$ on a measurable space $(T, \mathcal{T})$ is said to be smooth iff there exists a sequence $\left(A_{n}\right)_{n \in \mathbb{N}} \subseteq \mathcal{T}$ such that

$$
t \rho t^{\prime} \text { iff } \forall n \in \mathbb{N}:\left[t \in A_{n} \Leftrightarrow t^{\prime} \in A_{n}\right] .
$$

We say that the sequence $\left(A_{n}\right)_{n \in \mathbb{N}}$ determines relation $\rho$.

Denote by $\mathcal{I}(\mathcal{T}, \rho)$ the $\sigma$-algebra of $\rho$-invariant measurable sets, thus

$$
\mathcal{I}(\mathcal{T}, \rho):=\{A \in \mathcal{T} \mid A \text { is } \rho-\text { invariant }\},
$$

where $A \subseteq T$ is called $\rho$-invariant iff $A=\bigcup\left\{[t]_{\rho} \mid t \in A\right\}$ holds, thus iff $t \in A$ and $t \rho t^{\prime}$ together imply $t^{\prime} \in A$. We will see that smooth equivalence relations are just the natural kind of equivalence relations compatible with the structure of stochastic relations.

Observing a stochastic system $K: X \rightsquigarrow Y$, pairs with equivalent behavior are identified. This leads to a pair $(\alpha, \beta)$ of equivalence relations on the inputs $X$ resp. the outputs $Y$ with the idea that equivalent inputs lead to equivalent outputs. While equivalent inputs can be described directly through $\alpha$, the equivalence of outputs requires a description on the level of measurable sets. We argue that a set $B \subseteq Y$ does not distinguish between equivalent outputs iff it is invariant under $\beta$, i.e., if $y \in B$ and $y \beta y^{\prime}$ together imply $y^{\prime} \in B$. This leads then naturally to the notion of a congruence:

Definition $2 A$ congruence $\mathrm{c}=(\alpha, \beta)$ for the stochastic relation $\mathrm{K}=(X, Y, K)$ is a pair of smooth equivalence relations $\alpha$ on $X$ and $\beta$ on $Y$ such that $K(x)(D)=K\left(x^{\prime}\right)(D)$ holds whenever $x \alpha x^{\prime}$ and $D$ is an $\beta$-invariant measurable subset of $Y$.

Denote for the equivalence relation $\rho$ on the analytic space $T$ by $T / \rho$ the set of equivalence classes, and let $\eta_{\rho}: t \mapsto[t]_{\rho}$ assign to each $t$ its class $[t]_{\rho}$; denote by $\mathcal{T} / \rho$ the final $\sigma$-algebra on $T / \rho$ with respect to the Borel sets on $T$ and the natural projection $\eta_{\rho}$.

Smooth equivalence relations arise in a natural fashion from kernels of measurable maps, as we will see in a moment. These relations enjoy the technically interesting property that the factor space $(T / \rho, \mathcal{B}(T) / \rho)$ for an analytic space $T$ and a smooth relation $\rho$ is an analytic space again, cf. [1, Corollary 3.3.5.2]. In particular, $\mathcal{B}(T / \rho)=\mathcal{B}(T) / \rho$ holds. It can be said a wee bit more. We define the kernel ker $(f)$ of a map $f: M \rightarrow N$ as

$$
\operatorname{ker}(f):=\left\{\left\langle m, m^{\prime}\right\rangle \in M \times M \mid f(m)=f\left(m^{\prime}\right)\right\} .
$$

It is clear that $\operatorname{ker}(f)$ is an equivalence relation on $M$.

Lemma 1 Let $S, T$ be analytic spaces, and assume that $f: S \rightarrow T$ is a surjective and Borel measurable map. Then $\operatorname{ker}(f)$ is smooth, and $f^{-1}[\mathcal{B}(T)]=\mathcal{I}(\mathcal{B}(S), \operatorname{ker}(f))$.

\section{Proof}


0 . Because $T$ is an analytic space, its Borel sets possess a countable generator $\left(A_{n}\right)_{n \in \mathbb{N}}$ which separates points (thus for two distinct elements of $T$ there exists $A_{n}$ which contains one but not the other). Consequently,

$$
\begin{aligned}
s \operatorname{ker}(f) s^{\prime} & \Leftrightarrow f(s)=f\left(s^{\prime}\right) \\
& \Leftrightarrow \forall n \in \mathbb{N}:\left[f(s) \in A_{n} \Leftrightarrow f\left(s^{\prime}\right) \in A_{n}\right] \\
& \Leftrightarrow \forall n \in \mathbb{N}:\left[s \in f^{-1}\left[A_{n}\right] \Leftrightarrow s^{\prime} \in f^{-1}\left[A_{n}\right]\right]
\end{aligned}
$$

1. Given $B \in \mathcal{I}(\mathcal{B}(S)$, $\operatorname{ker}(f))$, we show first that $f^{-1}[f[B]]=B$ holds. In fact, $B \subseteq$ $f^{-1}[f[B]]$ is always true. Let $s \in f^{-1}[f[B]]$, thus $f(s)=f\left(s^{\prime}\right)$ for some $s^{\prime} \in B$. Since $B$ is $\operatorname{ker}(f)$-invariant, this implies $s \in B$, accounting for the other inclusion.

2. Let again $B \in \mathcal{I}(\mathcal{B}(S)$, $\operatorname{ker}(f))$, then $f[B] \subseteq T$ is analytic. We claim that $f[S \backslash B]=$ $T \backslash f[B]$ holds. For, if $t \in f[S \backslash B]$, we can find $s \notin B$ with $f(s)=t$. Assuming that $t=f\left(s^{\prime}\right)$ for some $s^{\prime} \in B$, we would infer that $s \in B$ due to the $\operatorname{ker}(f)$-invariance of $B$, and since $\left\langle s, s^{\prime}\right\rangle \in \operatorname{ker}(f)$. This is a contradiction. This settles the non-trivial inclusion. From the representation just established we see that $T \backslash f[B]$ is analytic, and from Souslin's Theorem [11, Theorem 4.4.3] we infer now that $f[B]$ is Borel in $T$.

3. It is clear that for each $C \in \mathcal{B}(T)$ its inverse image $f^{-1}[C]$ under $f$ is a Borel set which is $\operatorname{ker}(f)$-invariant. On the other hand, if $B \in \mathcal{I}(\mathcal{B}(S), \operatorname{ker}(f))$, we write $B=f^{-1}[f[B]]$ by part 1 , and $f[B] \in \mathcal{B}(T)$ by part 2 . This implies the desired equality.

As a by-product we obtain a characterization of $\rho$-invariant Borel sets in analytic spaces through the generating sequence $\left(A_{n}\right)_{n \in \mathbb{N}}$; this result is well-known for Polish spaces, cp. [11, Lemma 5.1.16]. As a consequence, we can characterize the $\rho$-invariant Borel set through the canonic projection.

Corollary 1 Let $T$ be an analytic space with a smooth equivalence relation $\rho$, then the $\rho$ invariant Borel sets of $T$ are exactly the inverse images of the canonic projection $\eta_{\rho}$, viz., $\mathcal{I}(\mathcal{B}(T), \rho)=\eta_{\rho}^{-1}[\mathcal{B}(T / \rho)]$ holds. Moreover, if $\rho$ is determined by the sequence $\left(A_{n}\right)_{n \in \mathbb{N}}$ of Borel sets $A_{n} \subseteq T$, then

$$
\mathcal{I}(\mathcal{B}(T), \rho)=\sigma\left(\left\{A_{n} \mid n \in \mathbb{N}\right\}\right) .
$$

Proof $1 . T / \rho$ is an analytic space, and $\eta_{\rho}: T \rightarrow T / \rho$ is surjective and onto. Thus the first assertion follows from Lemma 1 upon observing that $\rho=\operatorname{ker}\left(\eta_{\rho}\right)$ holds.

2. Let $B \in \mathcal{B}(T / \rho)$ be a Borel set in $T / \rho$. Plainly,

$$
\left.B=\bigcup\left\{\{t]_{\rho}\right\} \mid[t]_{\rho} \in B\right\},
$$

so it is enough to show that each $\left\{[t]_{\rho}\right\}$ constitutes an atom in $\sigma\left(\left\{\eta_{\rho}\left[A_{n}\right] \mid n \in \mathbb{N}\right\}\right)$.

Granted that, we can argue as follows: The Blackwell-Mackey-Theorem [11, Theorem 4.5.7] implies that

$$
\mathcal{B}(T / \rho)=\sigma\left(\left\{\eta_{\rho}\left[A_{n}\right] \mid n \in \mathbb{N}\right\}\right)
$$

holds, thus $C \in \mathcal{I}(\mathcal{B}(T), \rho)$ iff $C=\eta_{\rho}^{-1}[B]$ for some

$$
B \in \eta_{\rho}^{-1}\left[\sigma\left(\left\{\eta_{\rho}\left[A_{n}\right] \mid n \in \mathbb{N}\right\}\right)\right]=\sigma\left(\left\{A_{n} \mid n \in \mathbb{N}\right\}\right) .
$$

3. It is easy to see that

$$
\bigcap\left\{\eta_{\rho}\left[A_{n}\right] \mid t \in A_{n}\right\} \cap \bigcap\left\{T / \rho \backslash \eta_{\rho}\left[A_{n}\right] \mid t \notin A_{n}\right\},
$$


contains the class $[t]_{\rho}$ as its only element, and that

$$
T / \rho \backslash \eta_{\rho}\left[A_{n}\right]=\eta_{\rho}\left[T \backslash A_{n}\right],
$$

because $A_{n}$ is $\rho$-invariant, cp. part 2 of the proof of Lemma 1. Thus the atom $\left\{[t]_{\rho}\right\}$ is a member of $\sigma\left(\left\{\eta_{\rho}\left[A_{n}\right] \mid n \in \mathbb{N}\right\}\right)$.

The next Corollary shows that kernels of morphisms and congruences are basically the same thing. Denote for the morphism $\mathrm{f}: \mathrm{K}_{1} \rightarrow \mathrm{K}_{2}$ with $\mathrm{f}=(\phi, \psi)$ its kernel ker $(\mathfrak{f})$ by the pair $(\operatorname{ker}(\phi), \operatorname{ker}(\psi))$.

Corollary 2 If $\mathrm{f}: \mathrm{K} \rightarrow \mathrm{K}^{\prime}$ is a morphism for the stochastic relations $\mathrm{K}$ and $\mathrm{K}^{\prime}$, then $\operatorname{ker}(\mathrm{f})$ is a congruence for $\mathrm{K}$.

Proof Let $\mathrm{K}=(X, Y, K)$ and $\mathrm{K}^{\prime}=\left(X^{\prime}, Y^{\prime}, K^{\prime}\right)$ with $\mathrm{f}=(\phi, \psi)$. Let $x \operatorname{ker}(\phi) x^{\prime}$ and $D \subseteq Y$ be a $\operatorname{ker}(\psi)$-invariant Borel subset of $Y$. Lemma 1 shows that $D=\psi^{-1}\left[D^{\prime}\right]$ for some Borel set $D^{\prime} \subseteq Y^{\prime}$. Thus

$$
\begin{aligned}
K(x)(D) & =K(x)\left(\psi^{-1}\left[D^{\prime}\right]\right) \\
& =(\mathfrak{S}(\psi) \circ K)(x)\left(D^{\prime}\right) \\
& =\left(K^{\prime} \circ \phi\right)(x)\left(D^{\prime}\right) \\
& =K(\phi(x))\left(D^{\prime}\right) \\
& =K\left(\phi\left(x^{\prime}\right)\right)\left(D^{\prime}\right) \\
& =K\left(x^{\prime}\right)(D),
\end{aligned}
$$

since $\mathbf{f}=(\phi, \psi)$ is a morphism.

This construction permits introducing factor objects. Let $\mathrm{c}=(\alpha, \beta)$ be a congruence on the stochastic relation $\mathrm{K}=(X, Y, K)$, and define

$$
K_{\alpha, \beta}\left([x]_{\alpha}\right)(D):=K(x)\left(\eta_{\beta}^{-1}[D]\right)
$$

for $x \in X, D \in \mathcal{B}(Y / \beta)$, then

$$
\mathrm{K} / \mathrm{c}:=\left(X / \alpha, Y / \beta, K_{\alpha, \beta}\right)
$$

is a stochastic relation, and

$$
\eta_{\mathrm{c}}:=\left(\eta_{\alpha}, \eta_{\beta}\right): \mathrm{K} \rightarrow \mathrm{K} / \mathrm{c}
$$

is a morphism [6, Proposition 3].

\section{Two Isomorphisms}

We will investigate the factor of a stochastic relation through a congruence. Two isomorphisms will be considered: factoring a factor space, and factoring a morphism. Before we tackle these questions, we investigate what happens on the level of the underlying analytic spaces.

Assume that $\rho$ is a smooth equivalence relation on the analytic space $T$, and that $\tau$ is a smooth equivalence on $T / \rho$. Define for $t, t^{\prime} \in T$

$$
t(\tau \bullet \rho) t^{\prime} \Leftrightarrow[t]_{\rho} \tau\left[t^{\prime}\right]_{\rho}
$$


Proposition 1 The equivalence relation $\tau \bullet \rho$ is smooth, and the analytic spaces $T / \tau \bullet \rho$ and $(T / \rho) / \tau$ are Borel isomorphic.

Proof 0 . Since $\tau$ is smooth, there exists a sequence $\left(A_{n}\right)_{n \in \mathbb{N}}$ of Borel sets $A_{n} \subseteq T / \rho$ which determines it. Then $\left(\eta_{\rho}^{-1}\left[A_{n}\right]_{n \in \mathbb{N}}\right)$ determines $\tau \bullet \rho$. Its members are by construction Borel sets in $T$.

1. Define $g_{\rho, \tau}\left([t]_{\tau \bullet \rho}\right):=\left[[t]_{\rho}\right]_{\tau}$, then $g_{\rho, \tau}: T / \tau \bullet \rho \rightarrow(T / \rho) / \tau$ is well-defined and turns out to be a bijection. The construction shows that $g_{\rho, \tau} \circ \eta_{\tau \bullet \rho}=\eta_{\tau} \circ \eta_{\rho}$ holds, putting $h_{\rho, \tau}:=g_{\rho, \tau}^{-1}$, we see that $\eta_{\tau \bullet \rho}=h_{\rho, \tau} \circ \eta_{\tau} \circ \eta_{\rho}$. This is also noted for later use.

2. Let $E \subseteq(T / \rho) / \tau$ be a Borel set, we need to show that $g_{\rho, \tau}^{-1}[E]$ is a Borel set in $T / \tau \bullet \rho$, equivalently, that $E_{0}:=\eta_{\tau \bullet \rho}^{-1}\left[g_{\rho, \tau}^{-1}[E]\right]$ is an $\tau \bullet \rho$-invariant Borel set in $T$ by Lemma 1 . But $E_{0}=\left(\eta_{\rho} \circ \eta_{\tau}\right)^{-1}[E]$, so that $E_{0}$ is a Borel set by the measurability of the projections, and this set is clearly $\tau \bullet \rho$-invariant. Thus we get the measurability of $E_{0}$ again from Lemma 1 . 3. Let $F \subseteq T / \tau \bullet \rho$ be a Borel set, hence $F_{0}:=\eta_{\tau \bullet \rho}^{-1}[F]$ is a Borel set in $T$, thus there exists a Borel set $F_{1} \subseteq(T / \rho) / \tau$, such that $F_{0}=\eta_{\rho}^{-1}\left[\eta_{\tau}^{-1}\left[F_{1}\right]\right]$ since $F_{0}$ is $\rho$-invariant. Hence $F_{1}=h_{\rho, \tau}^{-1}[F]$, so $h_{\rho, \tau}$ is measurable, establishing the claim.

Now fix a stochastic relation $\mathrm{K}=(X, Y, K)$, and let $\mathrm{c}=(\rho, \tau)$ be a congruence on $\mathrm{K}$. Assume that $\mathbf{d}=(\kappa, \lambda)$ is a congruence of $\mathrm{K} / \mathrm{c}$. Define $\mathbf{d} \bullet \mathbf{c}:=(\kappa \bullet \rho, \lambda \bullet \tau)$

Proposition $2 \mathrm{~d} \bullet \mathrm{c}$ is a congruence on $\mathrm{K}$, and $\mathrm{K} / \mathrm{d} \bullet \mathrm{c}$ is isomorphic to $(\mathrm{K} / \mathrm{c}) / \mathrm{d}$

Proof 1. The first assertion follows from Corollary 2 together with the observation that $(\kappa \bullet \rho, \lambda \bullet \tau)=\left(\operatorname{ker}\left(\eta_{\kappa} \circ \eta_{\rho}\right), \operatorname{ker}\left(\eta_{\lambda} \circ \eta_{\tau}\right)\right)$ holds.

2. Construct the Borel isomorphisms $g_{\rho, \kappa}: X / \kappa \bullet \rho \rightarrow(X / \rho) / \kappa$ and $g_{\tau, \lambda}: Y / \lambda \bullet \tau \rightarrow(Y / \tau) / \lambda$ with their respective inverses $h_{\rho, \kappa}$ and $h_{\tau, \lambda}$ as in the proof of Proposition 1. We show that the inner and the outer diagram

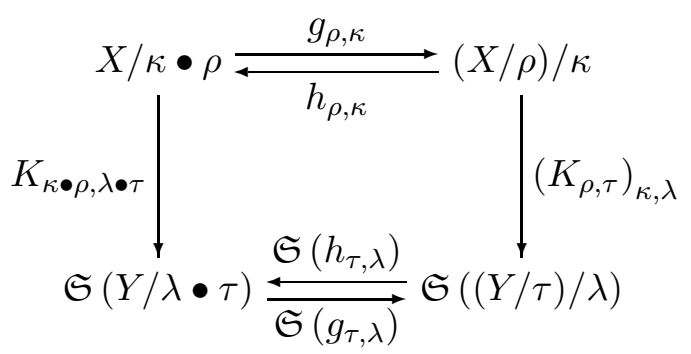

both commute.

3. Let $B \in \mathcal{B}((Y / \tau) / \lambda)$, a Borel set in $(Y / \tau) / \lambda$, then

$$
\begin{aligned}
K_{\kappa \bullet \rho, \lambda \bullet \tau}\left([x]_{\kappa \bullet \rho}\right)\left(g_{\tau, \lambda}^{-1}[B]\right) & =K(x)\left(\eta_{\lambda \bullet \tau}^{-1}\left[g_{\tau, \lambda}^{-1}[B]\right]\right) \\
& =K(x)\left(\eta_{\tau}^{-1}\left[\eta_{\lambda}^{-1}[B]\right]\right) \\
& =K_{\rho, \tau}\left([x]_{\rho}\right)\left(\eta_{\lambda}^{-1}[B]\right) \\
& =\left(K_{\rho, \tau}\right)_{\kappa, \lambda}\left(g_{\rho, \kappa}\left([x]_{\rho}\right)\right)(B),
\end{aligned}
$$

because $g_{\tau, \lambda} \circ \eta_{\lambda \bullet \tau}=\eta_{\lambda} \circ \eta_{\tau}$. Thus the outer diagram commutes. This implies that

$$
\mathrm{g}:=\left(g_{\rho, \kappa}, g_{\beta, \tau}\right): \mathrm{K} / \mathrm{d} \bullet \mathrm{c} \rightarrow(\mathrm{K} / \mathrm{c}) / \mathrm{d}
$$


is a morphism.

4. Suppose that $G \in \mathcal{B}(Y / \lambda \bullet \tau)$ is a Borel set, then

$$
\begin{aligned}
K_{\kappa \bullet \rho, \lambda \bullet \tau}\left(h_{\rho, \kappa}\left(\left[[x]_{\rho}\right]_{\kappa}\right)(G)\right. & =K_{\kappa \bullet \rho, \lambda \bullet \tau}\left([x]_{\kappa \bullet \rho}\right)(G) \\
& =K(x)\left(\eta_{\lambda \bullet \tau}^{-1}[G]\right) \\
& =K_{\rho, \tau}\left([x]_{\rho}\right)\left(\eta_{\lambda}^{-1}\left[h_{\tau, \lambda}^{-1}[G]\right]\right) \\
& =\left(K_{\rho, \tau}\right)_{\kappa, \lambda}\left(\left[[x]_{\rho}\right]_{\lambda}\right)\left(h_{\beta, \tau}^{-1}[G]\right)
\end{aligned}
$$

This is so since $\eta_{\lambda \bullet \tau}=h_{\tau, \lambda} \circ \eta_{\lambda} \circ \eta_{\tau}$ holds (see the proof of Proposition 1). Thus the inner diagram commutes. This implies that

$$
\mathrm{h}:=\left(h_{\rho, \kappa}, h_{\beta, \tau}\right):(\mathrm{K} / \mathrm{c}) / \mathrm{d} \rightarrow \mathrm{K} / \mathrm{d} \bullet \mathrm{c}
$$

is a morphism, and $\mathrm{h}$ is plainly left- and right inverse to $\mathrm{g}$.

Factoring a stochastic relation with a congruence entails identifying inputs resp. outputs that have been observed as representing identical behavior. Proposition 2 says then that identifying identical behavior in observing the factor system amounts to a system that can also be obtained through a single observational step from the original system. This means that there are no arbitrary long chains of factor systems which could not have been obtained directly from the original system, or, that factoring does not change the fundamental behavior of a system (after all, a system is bisimilar to its factor systems, bisimilarity requesting the existence of a span of morphisms, cp. $[2,4])$.

Algebraically, this proposition is quite similar to the well known Second Isomorphism Theorem of Group Theory, cp. [9, $\S$ I.4]: Factoring the quotient of a normal subgroup gives a group isomorphic to a factor. A similar but slightly stronger construction for coalgebras is carried out by Rutten [10, Theorem 7.4] in the context of bisimulation relations for coalgebras. Proposition 2 and Rutten's Theorem are not comparable directly, however, since the functor underlying the coalgebra is assumed to have weak pullbacks (which is no realistic assumption for stochastic relations, see [4, Remark 2]), and since the relationship between bisimulations and congruences is slightly less involved in the coalgebraic case.

Let $(\alpha, \beta)$ and $\left(\alpha^{\prime}, \beta^{\prime}\right)$ be pairs of equivalence relations, and define $(\alpha, \beta) \preceq\left(\alpha^{\prime}, \beta^{\prime}\right)$ iff $\alpha$ refines $\alpha^{\prime}$ and $\beta$ refines $\beta^{\prime}$ simultaneously, formally:

$$
(\alpha, \beta) \preceq\left(\alpha^{\prime}, \beta^{\prime}\right) \Leftrightarrow \alpha \subseteq \alpha^{\prime} \text { and } \beta \subseteq \beta^{\prime}
$$

It is clear that $\mathrm{c} \preceq \mathrm{d} \bullet \mathrm{c}$ for each congruence $\mathrm{d}$.

Proposition 3 Assume that $\mathrm{f}: \mathrm{K} \rightarrow \mathrm{K}^{\prime}$ is a morphism, and let $\mathrm{c}$ be a congruence on $\mathrm{K}$ such that $\mathrm{c} \preceq \operatorname{ker}(\mathrm{f})$. Then there exists a unique morphism $\mathrm{f}_{\mathrm{c}}: \mathrm{K} / \mathrm{c} \rightarrow \mathrm{K}^{\prime}$ with $\mathrm{f}=\mathrm{f}_{\mathrm{c}} \circ \eta_{\mathrm{c}}$

Proof 1 . Let $\mathrm{K}=(X, Y, K), \mathrm{K}^{\prime}=\left(X^{\prime}, Y^{\prime}, K^{\prime}\right)$ with $\phi: X \rightarrow X^{\prime}, \psi: Y \rightarrow Y^{\prime}$ constituting morphism $\mathrm{f}$, and $\mathrm{c}=(\alpha, \beta)$. Because $\alpha \subseteq \operatorname{ker}(\phi), \beta \subseteq \operatorname{ker}(\psi)$, the maps

$$
\begin{aligned}
& \phi_{\alpha}\left([x]_{\alpha}\right):=\phi(x), \\
& \psi_{\beta}\left([y]_{\beta}\right):=\psi(y)
\end{aligned}
$$


are well defined. Since $\phi$ is $\mathcal{B}(X)-\mathcal{B}\left(X^{\prime}\right)$-measurable, and since $\mathcal{B}(X) / \alpha$ is the final $\sigma$ algebra on $X / \alpha$ with respect to $\eta_{\alpha}, \mathcal{B}(X) / \alpha-\mathcal{B}\left(X^{\prime}\right)$-measurability of $\phi_{\alpha}$ is inferred. A similar argument is used for $\psi_{\beta}$. Clearly, these maps are onto.

2. It remains to show that $\mathrm{f}_{\mathrm{c}}:=\left(\phi_{\alpha}, \psi_{\beta}\right)$ is a morphism. In fact, let $D^{\prime} \subseteq Y^{\prime}$ be a Borel set, then

$$
\begin{aligned}
K^{\prime}\left(\phi_{\alpha}\left([x]_{\alpha}\right)\right)\left(D^{\prime}\right) & =K^{\prime}(\phi(x))\left(D^{\prime}\right) \\
& =K(x)\left(\psi^{-1}\left[D^{\prime}\right]\right) \\
& =K_{\alpha, \beta}\left([x]_{\alpha}\right)\left(\psi_{\beta}^{-1}\left[D^{\prime}\right]\right) \\
& =\left(\mathfrak{S}\left(\psi_{\beta}\right) \circ K_{\alpha, \beta}\right)\left([x]_{\alpha}\right)\left(D^{\prime}\right)
\end{aligned}
$$

because $\psi^{-1}\left[D^{\prime}\right]=\eta_{\beta}^{-1}\left[\psi_{\beta}^{-1}\left[D^{\prime}\right]\right]$, and because $\left(\eta_{\alpha}, \eta_{\beta}\right)$ is a morphism. Consequently, the equality $K^{\prime} \circ \phi_{\alpha}=\mathfrak{S}\left(\psi_{\beta}\right) \circ K_{\alpha, \beta}$ has been established. Uniqueness is obvious.

Corollary 3 Assume that $\mathrm{f}: \mathrm{K} \rightarrow \mathrm{K}^{\prime}$ is a morphism. Then there exists a unique isomorphism $\mathrm{f}^{\sharp}: \mathrm{K} / \operatorname{ker}(\mathrm{f}) \rightarrow \mathrm{K}^{\prime}$ with $\mathrm{f}=\mathrm{f}^{\sharp} \circ \eta_{\operatorname{ker}(\mathrm{f})}$.

Proof Define $f^{\sharp}:=f_{\text {ker }(f)}$, then the maps constituting this morphism are bijective Borel maps, so by [11, Proposition 4.5.1] they are Borel isomorphisms. The equations establishing the morphism property for $\mathrm{f}_{\mathrm{ker}(\mathrm{f})}$ show that the inverses also constitute a morphism.

Corollary 4 Let $\mathrm{c}$ and $\mathrm{d}$ be congruences on $\mathrm{K}$, then the following statement are equivalent:

1. $c \preceq d$

2. $\mathrm{d}=\mathrm{e} \bullet \mathrm{c}$ for some congruence e on $\mathrm{K}$.

Proof The implication $(2) \Rightarrow(1)$ is obvious. Assume that $c \preceq d=\operatorname{ker}\left(\eta_{d}\right)$ holds. Then the assertion follows from Proposition 3 together with Corollary 2.

This property is somewhat surprising in that it relates the refinement of congruences to factor spaces. If $\mathrm{c}$ is finer than $\mathrm{d}$, then congruence $\mathrm{d}$ can be obtained through observing and factoring the behavior in the factor system for $\mathrm{c}$ (so that not the original system has to be observed but rather a simplified one).

\section{Conclusion}

Let's wrap things up by considering labelled Markov transition systems. Fix an analytic state space $S$ and a countable set $A$ of actions. Then $\mathbb{K}:=\left(S,\left(k_{a}\right)_{a \in A}\right)$ is called a labelled Markov transition process iff $k_{a}: S \rightsquigarrow S$ is a stochastic relation for each $a \in A$, see e.g. [2, 4]. The surjective Borel map $\phi: S \rightarrow S^{\prime}$ constitutes a morphism $\left(S,\left(k_{a}\right)_{a \in A}\right) \rightarrow\left(S^{\prime},\left(\ell_{a}\right)_{a \in A}\right)$ iff $\ell_{a} \circ \phi=\mathfrak{S}(\phi) \circ k_{a}$ holds for each action $a \in A$, or, equivalently, if $(\phi, \phi):\left(S, S, k_{a}\right) \rightarrow\left(S^{\prime}, S^{\prime}, \ell_{a}\right)$ holds in Stoch for each $a \in A$. Similarly, a smooth equivalence relation $\gamma$ on $S$ is said to be a congruence on $\mathbb{K}$ iff

$$
s \gamma s^{\prime} \Rightarrow \forall C \in \mathcal{I}(\mathcal{B}(S), \gamma): k_{a}(s)(C)=k_{a}\left(s^{\prime}\right)(C)
$$

holds for each $a \in A$. This generalizes the relation defined through the Hennessy-Milner equivalence for a simple negation free modal logic with $\langle a\rangle_{q}$ as diamonds for $a \in A$ and rational $q$ that is investigated in $[2,4]$. 
It is clear that a congruence $\gamma$ gives rise to a factor system $\mathbb{K} / \gamma:=\left(S,\left(k_{a, \gamma}\right)_{a \in A}\right)$, and that the kernel of a morphism is a congruence. We obtain then from the discussion above

1. If $\gamma$ is a congruence on $\mathbb{K}$, and $\delta$ is a congruence on $\mathbb{K} / \gamma$, then $(\mathbb{K} / \gamma) / \delta$ is isomorphic to $\mathbb{K} / \delta \bullet \gamma$,

2. Each morphism $\phi: \mathbb{K} \rightarrow \mathbb{L}$ factors uniquely through $\mathbb{K} / \operatorname{ker}(\phi)$, and $\mathbb{K} / \operatorname{ker}(\phi)$ is isomorphic to $\mathbb{L}$.

In this way, investigating congruences for stochastic relations turns out to be a fruitful endeavor for labelled Markov transition systems.

\section{References}

[1] W. Arveson. An Invitation to $C^{*}$-Algebra, volume 39 of Graduate Texts in Mathematics. Springer-Verlag, New York, 1976.

[2] J. Desharnais, A. Edalat, and P. Panangaden. Bisimulation of labelled Markov-processes. Information and Computation, 179(2):163 - 193, 2002.

[3] E.-E. Doberkat. Pipelines: Modelling a software architecture through relations. Acta Informatica, 40:37 - 79, 2003.

[4] E.-E. Doberkat. Semi-pullbacks and bisimulations in categories of stochastic relations. In J. C. M. Baeten, J. K. Lenstra, J. Parrow, and G. J. Woeginger, editors, Proc. ICALP'03, volume 2719 of Lecture Notes in Computer Science, pages 996 - 1007, Berlin, July 2003. Springer-Verlag.

[5] E.-E. Doberkat. Semi-pullbacks for stochastic relations over analytic spaces. Technical Report 142, Chair for Software-Technology, University of Dortmund, October 2003.

[6] E.-E. Doberkat. Stochastic relations interpreting modal logic. Technical Report 144, Chair for Software-Technology, University of Dortmund, October 2003.

[7] M. Giry. A categorical approach to probability theory. In Categorical Aspects of Topology and Analysis, volume 915 of Lecture Notes in Mathematics, pages 68 - 85, Berlin, 1981. Springer-Verlag.

[8] S. Mac Lane. Categories for the Working Mathematician. Number 5 in Graduate Texts in Mathematics. Springer-Verlag, Berlin, 2 edition, 1997.

[9] S. Lang. Algebra. Addison-Wesley, Reading, Mass., 1965.

[10] J. J. M. M. Rutten. Universal coalgebra: a theory of systems. Theoretical Computer Science, 249(1):3 - 80, 2000. Special issue on modern algebra and its applications.

[11] S. M. Srivastava. A Course on Borel Sets. Number 180 in Graduate Texts in Mathematics. Springer-Verlag, Berlin, 1998. 
/99/ T. Bühren, M. Cakir, E. Can, A. Dombrowski, G. Geist, V. Gruhn, M. Gürgrn, S. Handschumacher, M. Heller, C. Lüer, D. Peters, G. Vollmer, U. Wellen, J. von Werne Endbericht der Projektgruppe eCCo (PG 315) Electronic Commerce in der Versicherungsbranche Beispielhafte Unterstützung verteilter Geschäftsprozesse Februar 1999

/100/ A. Fronk, J. Pleumann, Der DoDL-Compiler August 1999

/101/ K. Alfert, E.-E. Doberkat, C. Kopka Towards Constructing a Flexible Multimedia Environment for Teaching the History of Art September 1999

/102/ E.-E. Doberkat An Note on a Categorial Semantics for ER-Models November 1999

/103/ Christoph Begall, Matthias Dorka, Adil Kassabi, Wilhelm Leibel, Sebastian Linz, Sascha Lüdecke, Andreas Schröder, Jens Schröder, Sebastian Schütte, Thomas Sparenberg, Christian Stücke, Martin Uebing, Klaus Alfert, Alexander Fronk, Ernst-Erich Doberkat Abschlußbericht der Projektgruppe PG-HEU (326) Oktober 1999

/104/ Corina Kopka Ein Vorgehensmodell für die Entwicklung multimedialer Lernsysteme März 2000

/105/ Stefan Austen, Wahid Bashirazad, Matthais Book, Traugott Dittmann, Bernhard Flechtker, Hassan Ghane, Stefan Göbel, Chris Haase, Christian Leifkes, Martin Mocker, Stefan Puls, Carsten Seidel, Volker Gruhn, Lothar Schöpe, Ursula Wellen

Zwischenbericht der Projektgruppe IPSI

April 2000

/106/ Ernst-Erich Doberkat

Die Hofzwerge - Ein kurzes Tutorium zur objektorientierten Modellierung

September 2000

/107/ Leonid Abelev, Carsten Brockmann, Pedro Calado, Michael Damatow, Michael Heinrichs, Oliver Kowalke, Daniel Link, Holger Lümkemann, Thorsten Niedzwetzki, Martin Otten, Michael Rittinghaus, Gerrit Rothmaier Volker Gruhn, Ursula Wellen

Zwischenbericht der Projektgruppe Palermo November 2000

/108/ Stefan Austen, Wahid Bashirazad, Matthais Book, Traugott Dittmann, Bernhard Flechtker, Hassan Ghane, Stefan Göbel, Chris Haase, Christian Leifkes, Martin Mocker, Stefan Puls, Carsten Seidel, Volker Gruhn, Lothar Schöpe, Ursula Wellen

Endbericht der Projektgruppe IPSI

Februar 2001

/109/ Leonid Abelev, Carsten Brockmann, Pedro Calado, Michael Damatow, Michael Heinrichs, Oliver Kowalke, Daniel Link, Holger Lümkemann, Thorsten Niedzwetzki, Martin Otten, Michael Rittinghaus, Gerrit Rothmaier Volker Gruhn, Ursula Wellen Zwischenbericht der Projektgruppe Palermo Februar 2001

/110/ Eugenio G. Omodeo, Ernst-Erich Doberkat Algebraic semantics of ER-models from the standpoint of map calculus. Part I: Static view März 2001

/111/ Ernst-Erich Doberkat An Architecture for a System of Mobile Agents März 2001 
/112/ Corina Kopka, Ursula Wellen

Development of a Software Production Process Model for Multimedia CAL Systems by Applying Process Landscaping

April 2001

/113/ Ernst-Erich Doberkat

The Converse of a Probabilistic Relation

Oktober 2002

/114/ Ernst-Erich Doberkat, Eugenio G. Omodeo

Algebraic semantics of ER-models in the context of the calculus of relations.

Part II: Dynamic view

Juli 2001

/115/ Volker Gruhn, Lothar Schöpe (Eds.)

Unterstützung von verteilten Softwareentwicklungsprozessen durch integrierte Planungs-, Workflow- und Groupware-

Ansätze

September 2001

/116/ Ernst-Erich Doberkat

The Demonic Product of Probabilistic Relations

September 2001

/117/ Klaus Alfert, Alexander Fronk, Frank Engelen

Experiences in 3-Dimensional Visualization of Java Class Relations

September 2001

/118/ Ernst-Erich Doberkat

The Hierarchical Refinement of Probabilistic Relations

November 2001

/119/ Markus Alvermann, Martin Ernst, Tamara Flatt, Urs Helmig, Thorsten Langer, Ingo Röpling, Clemens Schäfer, Nikolai Schreier, Olga Shtern

Ursula Wellen, Dirk Peters, Volker Gruhn

Project Group Chairware Intermediate Report

November 2001

/120/ Volker Gruhn, Ursula Wellen

Autonomies in a Software Process Landscape

Januar 2002

/121/ Ernst-Erich Doberkat, Gregor Engels (Hrsg.)

Ergebnisbericht des Jahres 2001

des Projektes "MuSofT - Multimedia in der SoftwareTechnik"

Februrar 2002

/122/ Ernst-Erich Doberkat, Gregor Engels, Jan Hendrik Hausmann, Mark Lohmann, Christof Veltmann

Anforderungen an eine eLearning-Plattform - Innovation und Integration -

April 2002

/123/ Ernst-Erich Doberkat

Pipes and Filters: Modelling a Software Architecture Through Relations

Juni 2002

/124/ Volker Gruhn, Lothar Schöpe Integration von Legacy-Systemen mit Eletronic Commerce Anwendungen Juni 2002

/125/ Ernst-Erich Doberkat

A Remark on A. Edalat's Paper Semi-Pullbacks and Bisimulations in Categories of Markov-Processes Juli 2002

/126/ Alexander Fronk

Towards the algebraic analysis of hyperlink structures

August 2002

/127/ Markus Alvermann, Martin Ernst, Tamara Flatt, Urs Helmig, Thorsten Langer

Ingo Röpling, Clemens Schäfer, Nikolai Schreier, Olga Shtern

Ursula Wellen, Dirk Peters, Volker Gruhn

Project Group Chairware Final Report

August 2002 
/128/ Timo Albert, Zahir Amiri, Dino Hasanbegovic, Narcisse Kemogne Kamdem,

Christian Kotthoff, Dennis Müller, Matthias Niggemeier, Andre Pavlenko, Stefan Pinschke,

Alireza Salemi, Bastian Schlich, Alexander Schmitz

Volker Gruhn, Lothar Schöpe, Ursula Wellen

Zwischenbericht der Projektgruppe Com42Bill (PG 411)

September 2002

/129/ Alexander Fronk

An Approach to Algebraic Semantics of Object-Oriented Languages

Oktober 2002

/130/ Ernst-Erich Doberkat

Semi-Pullbacks and Bisimulations in Categories of Stochastic Relations

November 2002

/131 Yalda Ariana, Oliver Effner, Marcel Gleis, Martin Krzysiak, Jens Lauert, Thomas Louis, Carsten Röttgers, Kai Schwaighofer,

Martin Testrot, Uwe Ulrich, Xingguang Yuan

Prof. Dr. Volker Gruhn, Sami Beydeda

Endbericht der PG nightshift:

Dokumentation der verteilten Geschäftsprozesse im FBI und Umsetzung von Teilen dieser Prozesse im Rahmen eines FBI-Intranets basierend auf WAP- und Java-Technologie

Februar 2003

/132/ Ernst-Erich Doberkat, Eugenio G. Omodeo

ER Modelling from First Relational Principles

Februar 2003

/133/ Klaus Alfert, Ernst-Erich Doberkat, Gregor Engels (Hrsg.)

Ergebnisbericht des Jahres 2002 des Projektes "MuSofT - Multimedia in der SoftwareTechnik" März 2003

/134/ Ernst-Erich Doberkat

Tracing Relations Probabilistically

März 2003

135/ Timo Albert, Zahir Amiri, Dino Hasanbegovic, Narcisse Kemogne Kamdem, Christian Kotthoff, Dennis Müller, Matthias Niggemeier,

Andre Pavlenko, Alireza Salemi,Bastian Schlich, Alexander Schmitz,

Volker Gruhn, Lothar Schöpe, Ursula Wellen

Endbericht der Projektgruppe Com42Bill (PG 411)

März 2003

/136/ Klaus Alfert

Vitruv: Specifying Temporal Aspects of Multimedia Presentations -

A Transformational Approach based on Intervals

April 2003

/137/ Klaus Alfert, Jörg Pleumann, Jens Schröder

A Framework for Lightweight Object-Oriented Design Tools

April 2003

/138/ K. Alfert, A. Fronk, Ch. Veltmann (Hrsg.)

Stefan Borggraefe, Leonore Brinker, Evgenij Golkov, Rafael Hosenberg, Bastian Krol, Daniel Mölle, Markus Niehammer, Ulf Schellbach, Oliver Szymanski, Tobias Wolf, Yue Zhang

Endbericht der Projektgruppe 415: Konzeption und Implementierung eines digitalen und hypermedialen Automobilcockpits (HyCop)

Mai 2003

/139/ Volker Gruhn, Malte Hülder, Sami Beydeda (Hrsg.)

Endbericht der Projektgruppe 409: Entwicklung von ortsbasierten Diensten für UMTS-Mobilfunkgeräte (mCube)

Mai 2003

/140/ Ernst-Erich Doberkat

Congruences for Stochastic Relations

Juli 2003 
/141/ Marion Kamphans, Sigrid Metz-Göckel, Anja Tigges

Wie Geschlechteraspekte in die digitalen Medien integriert werden können - das BMBF-Projekt „MuSofT“

September 2003

/142/ Ernst-Erich Doberkat

Semi-Pullbacks for Stochastic Relations over Analytic Spaces

Oktober 2003

/143/ Volker Gruhn, Lothar Schöpe (Hrsg.)

1. Workshop des Verbundforschungsprojektes Mobile Spedition im Web - SpiW

Oktober 2002

/144/ Ernst-Erich Doberkat

Stochastic Relations Interpreting Modal Logic

Oktober 2003

/145/ Alexander Fronk, Ernst-Erich Doberkat, Johannes Bergemann, Ulrich-Walter Gans

Ein interdisziplinäres methodisches Vorgehen zur Gestaltung webbasierter Studieneinheiten für die Altertumswissenschaften

November 2003

/146/ Ernst-Erich Doberkat

Factoring Stochastic Relations

Januar 2004 